\title{
Simultaneous Attainability of the Central Exponents of Small-Dimensional Linear Hamiltonian Systems
}

\author{
T. V. Salova \\ Moscow State University, Moscow, Russia \\ e-mail:m_message@mail.ru
}

Received June 30, 2014

\begin{abstract}
We claim that the upper and lower central exponents of linear Hamiltonian systems of second and fourth orders are simultaneously attainable under uniformly small and infinitesimal Hamiltonian perturbations.
\end{abstract}

DOI: $10.1134 /$ S0012266114100188

Let $J$ be an orthogonal skew-symmetric operator on the Euclidean space $\mathbb{R}^{2 n}$; i.e., $J^{-1}=$ $J^{*}=-J$. A system of the form

$$
\dot{x}=A(t) x, \quad x \in \mathbb{R}^{2 n}, \quad t \in \mathbb{R}^{+} \equiv[0, \infty),
$$

with bounded piecewise continuous operator function $A$ is called a linear Hamiltonian system if the operator $J A(t)$ is symmetric for each $t \in \mathbb{R}^{+}$. We equip the linear space $\mathcal{H}^{2 n}$ of all such systems identified with the corresponding functions $A$ with the uniform topology on the half-line $t \in \mathbb{R}^{+}$, which is given by the norm $\|A\|=\sup _{t \in \mathbb{R}^{+}}|A(t)|$, where $|A(t)|=\sup _{|x|=1}|A(t) x|$.

To each system $A \in \mathcal{H}^{2 n}$, we assign its Lyapunov exponents $\lambda_{1}(A) \leq \cdots \leq \lambda_{2 n}(A)$ and upper and lower central exponents [1, pp. 115-117] $\Omega(A)$ and $\omega(A)$, which are treated as functionals on the space $\mathcal{H}^{2 n}$. In addition, by $\mathcal{H}_{\varepsilon}(A)$ or $\mathcal{H}_{0}(A)$ we denote the sets of all systems $B \in \mathcal{H}^{2 n}$ satisfying the condition $\|B-A\|<\varepsilon$ or $\lim _{t \rightarrow \infty}|B(t)-A(t)|=0$, respectively.

Theorem 1. If $n=1,2$, then for each $A \in \mathcal{H}^{2 n}$, there exists a system $B \in \mathcal{H}_{0}(A)$ satisfying the relations $\lambda_{1}(B)=\omega(A)$ and $\lambda_{2 n}(B)=\Omega(A)$.

Theorem 1 implies the following assertion.

Theorem 2. If $n=1,2$, then for any system $A \in \mathcal{H}^{2 n}$ and $\varepsilon>0$, there exists a system $B \in \mathcal{H}_{\varepsilon}(A)$ satisfying the inequalities $\lambda_{1}(B)<\omega(A)+\varepsilon$ and $\lambda_{2 n}(B)>\Omega(A)-\varepsilon$.

Remark. Theorem 2 for $n=1$ was announced in [2].

The results of the present paper were reported at the seminar on qualitative theory of differential equations at Moscow State University on October 24, 2014.

\section{REFERENCES}

1. Bylov, B.F., Vinograd, R.E., Grobman, D.M., and Nemytskii, V.V., Teoriya pokazatelei Lyapunova i ee prilozheniya $k$ voprosam ustoichivosti (Theory of Lyapunov Exponents and Its Applications to Stability Problems), Moscow: Nauka, 1966.

2. Salova, T.V., On the Simultaneous Attainability of the Central Exponents of Two-Dimensional Hamiltonian Systems, Differ. Uravn., 2006, vol. 42, no. 6, pp. 854-855. 